\title{
An Efficient Skin Cancer Diagnostic System Using Bendlet Transform and Support Vector Machine
}

\author{
POOVIZHI S \& GANESH BABU TR
}

\begin{abstract}
Skin is the outermost and largest organ of the human body that protects us from the external agents. Among the various types of diseases affecting the skin, melanoma (skin cancer) is the most dangerous and deadliest disease. Though it is one of the dangerous forms of cancer, it has a high survival rate if and only if it is diagnosed at the earliest. In this study, skin cancer classification (SCC) system is developed using dermoscopic images. It is considered as a classification problem with the help of Bendlet Transform (BT) as features and Support Vector Machine (SVM) as a classifier. First, the unwanted information's such as hair and noises are removed using median filtering approach. Then, directional representation based feature extraction system that precisely classifies curvature, location and orientation is employed. Finally, two SVM classifiers are designed for the classification. The performance of the SCC system based on Bendlet is superior to other image representation systems such as Wavelets, Curvelets, Contourlets and Shearlets.
\end{abstract}

Key words: Bendlet Transform, Medical image classification, Multi-resolution analysis, Skin cancer, Support vector machine.

\section{INTRODUCTION}

Nowadays, the incidence and mortality rate of skin cancer increasing worldwide. Hence, an automated SCC system is required for early detection and prevention of skin cancer. Dermoscopic imaging is the primary technique for the diagnosis of skin cancer. Ruiz et al. (2011) developed a decision support system for SCC. It uses ABCD (Asymmetry, Border, Colour and Diameter) rule with many descriptors such as modification ratio, anisotropy, sharpness variation, and roundness and components average of the colour. Then, three classifiers that were based on k-Nearest Neighbour $(\mathrm{kNN})$, Bayesian and Multilayer perceptron were employed for the classification using the selected features. Deep Neural networks based SCC system was developed by Esteva et al. (2017).
Only the biopsy-proven images were used to train and test the Google Inception architecture.

Texture and colour features based SCC system was implemented by Almansour et al. (2016). Colour features were extracted from different colour spaces such as $R G B, Y_{b} C_{r}$ CIE Lab and HSV. Texture features by employing local binary pattern and grey level co-occurrence matrix techniques were extracted. Finally, SVM classifier classifies the dermoscopic images. A combination of three different features was used for SCC by Nasir et al. (2018). Colour features from four colour spaces, shape features by the histogram of oriented gradients and fractal features were combined. From the combination, dominant features were selected and classified by the use of SVM classifier. 
ABCD rule was applied for the SCC system by Ozkan \& Koklu (2017). It uses four different classifiers such as kNN, SVM, decision tree and Artificial Neural Network (ANN) to classify the dermoscopic images. The various parameters of $A B C D$ rule were determined by various image processing approaches such as de-noising, segmentation, enhancement (Zaqout 2016). Then, a total dermoscopic score was computed and used for the classification. Different combination of features from $A B C D$ rule was analyzed by Ma et al. (2017) for SCC using SVM classifier. ABCD rule with a 7-point checklist (De vita et al. 2012) was used for the diagnosis of skin cancer.

Sonia(2016) developed Contourlet Transform based SCC system. It uses a non-sub-sampled version of the transform for feature extraction and Bayesian classifier. 10 fold cross-validation was used for performance analysis. Wavelet transform based SCC system was developed by Jain et al. (2012). The approximation coefficients from the decomposed dermoscopic images were given to the probabilistic neural network. Wavelet and Curvelet transform based SCC system were described by Abu mahmoud et al. (2013). Statistical features were extracted from the sub-band coefficients and then the feature space dimension was reduced by the principal component analysis. It uses ANN for the classification with a back propagation algorithm.

In this paper, a SCC system with dermoscopic images by the use of BT and SVM classifier is presented. The organization of the rest of this article is as follows: The definition and details about the SCC system with BT features and SVM classifier are discussed in Materials and Methods. It also gives the details about the materials used in this system. The results obtained by the SCC system are presented in Results and Discussions and finally conclusion of this study is given.

\section{MATERIALS AND METHODS}

The SCC system consists of three different modules; preprocessing, feature or information extraction and classification. The extracted features or information from the dermoscopic images affect the overall performance if they contain redundant data such as noise and hair. Hence, the unwanted information is removed using median filtering approach in the preprocessing module. The preprocessing step should preserve edges and curves in dermoscopic images while removing noises. The non linear filtering approach has potential improvement over linear filter (Arias-Castro \& Donoho 2009) where the preservation of edges and curves are of critical importance in medical image analysis. Hence, median filtering is used in this study which not only removes the noises but also hairs in the dermoscopic images. In the feature extraction module, the preprocessed dermoscopic image is represented by BT and then the energies of sub-band features are extracted. In the classification module, the extracted features are classified using SVM classifier. The framework of the proposed skin cancer diagnostic system is shown in Figure 1.

\section{BT based feature extraction}

Medical images consist of regions with different kinds of tissues which are separated by smooth curves. These smooth curves provide various information about the image and are defined by multiple functions i.e., piecewise in nature. Hence, the extraction of these curves helps to classify the images. To extract the curves, different multi-resolution analysis is being used in the last years. The directional representation systems (Curvelet (Donoho et al. 2005), Contourlet (Do \& Vetterli 2005), and Shearlet (Lim 2010)) differ from regular wavelets (Mallat 2008) where the degree of directions or orientation varies 


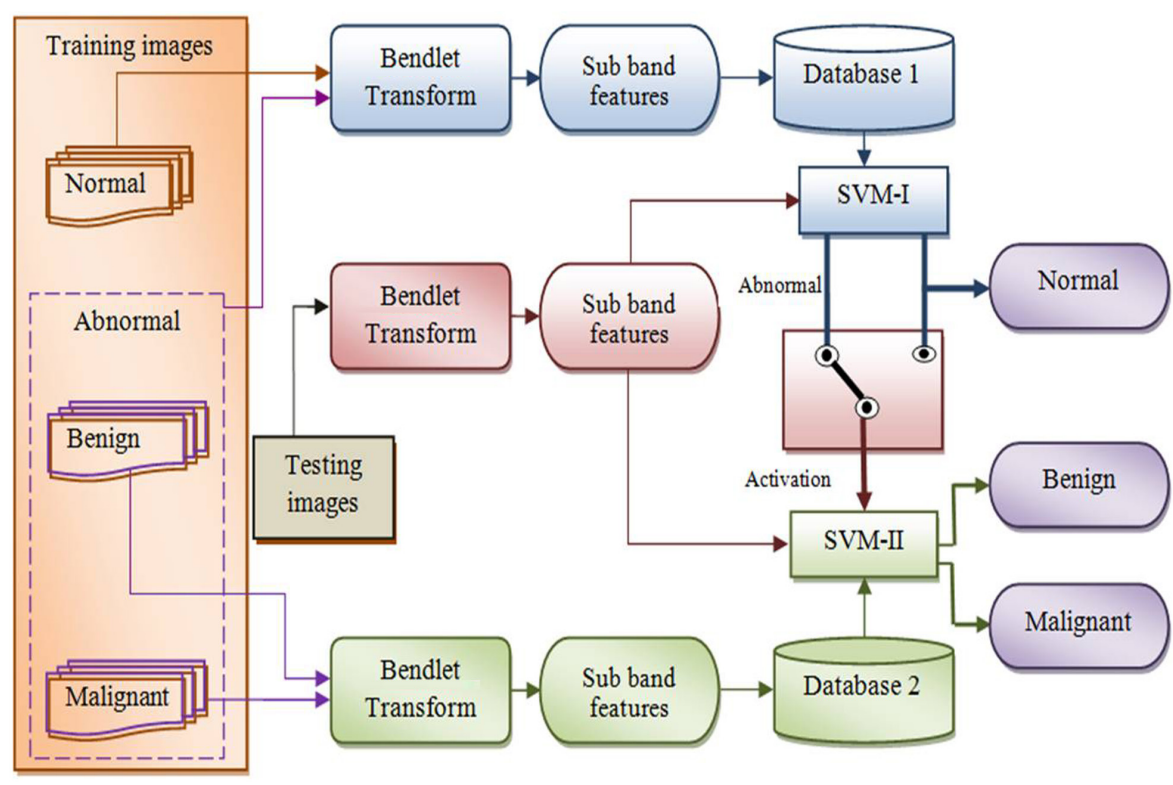

\author{
Figure 1. BT and SVM based \\ SCC system.
}

with the level of decomposition. This property locates the boundary curves precisely and also gives many directional information. One of the advantages of Shearlets is that it detects the nonsmooth corner points. However, the drawback of existing systems is that they cannot classify the curvature precisely. This can be overcome by the BT with an additional parameter in Shearlets called bending. The construction of BT from Shearlets is as follows:

Shearlet Transform ST $(\psi)$ can be written as

$S T(\psi)=T_{t} D_{A_{a}} D_{S_{s}} \psi$

where $T_{t}$ is the translation operator, $D_{A_{a}}$ is the dilation and $D_{S_{s}}$ is the shearing operator. The operators T and D are defined by Lim (2010),

$T_{t} \psi(x)=\psi(x-t)$

$D_{M} \psi(x)=|\operatorname{det} M|^{-\frac{1}{2}} \psi\left(M^{-1} x\right)$

The definition of scaling matrix and the shearing matrix are given below:

$$
A_{a}=\left(\begin{array}{cc}
a & 0 \\
0 & a^{\frac{1}{2}}
\end{array}\right) \text { where } a>0
$$

$S_{s}=\left(\begin{array}{ll}1 & s \\ 0 & 1\end{array}\right)$ where s is an integer

The scaling operator in (4) is called parabolic scaling (Lessig et al. 2017). The main difference of BT with Shearlet transform lies in the scaling operation where the parabolic scaling is replaced by $\alpha$ the scaling method. It is defined by Lessig et al. (2017),

$$
A_{a, \alpha}=\left(\begin{array}{cc}
a & 0 \\
0 & a^{\alpha}
\end{array}\right) \text { where } a>0 \text { and } \alpha \in[0,1]
$$

where the scaling anisotropy is defined by the $\alpha$ parameter (Lessig et al. 2017). With this $\alpha$ scaling method, the decay rate of bendlets provides more accurate curvature and directions of different regions in an image. More information about different image representations system can be found in Wavelets (Mallat 2008), Curvelet (Donoho et al. 2005), Contourlet (Do \& Vetterli 2005), Shearlet (Lim 2010) and Bendlet (Lessig et al. 2017).

After representing the dermoscopic image by the image representation system, characterizing textures in each sub-image is very important. This step not only reduces the feature space 
but also provides important information that could be used for accurate classification. Many image classification systems such as texture classification (Wang \& Yong 2008), mammogram classification (Ali \& Janet 2013), and fundus image classification (Selvathi et al. 2018) use the energy distribution of a sub-image as features. In this study, the mean of the magnitude of each BT sub-image is extracted for the SCC system. It is defined by

Energy distribution $=\frac{1}{R C} \sum_{i=1}^{R} \sum_{j=1}^{C}\left|B T_{\text {sub-image }}(i, j)\right|$

where $\left\{\left(t_{i}, c_{i}\right), i=1,2,3, \ldots N\right\}$ represents the subimage of size $\mathrm{R} \times \mathrm{C}$. Sub-image coordinates are represented by $(i, j)$.

\section{SVM Classifier}

The classification task is the final stage of BT and SVM based SCC system where the dermoscopic image is labeled into one of the predefined class. This task operates in two phases; training and testing. In the former phase, the association between two classes and outcomes are established whereas in the later stage the established or trained model is used to classify the testing data.

SVM classifier classifies the given data or feature by constructing a hyperplane with maximum margin. Let us consider the linear discriminant function $O$ with testing data $t$ and training data $T$ (Erasto 2001)

$$
O(t)=w^{T} t+b
$$

where $w$ and $b$ are the weight and bias value computed by using $T=\left\{\left(t_{i}, c_{i}\right), i=1,2,3, \ldots N\right\}$ respectively. The optimal separating hyperplane using these parameters can be written (Erasto 2001) as

$$
\min _{w, b} \frac{1}{2}\|w\|^{2}+C \sum_{i=1}^{n} \xi_{i}
$$

subject $b \quad c_{i} O\left(t_{i}\right) \geq 1-\xi i$, and $\xi i \geq 0, i=1,2, \ldots . n$. where $C$ is the user defined parameter which controls the trade-off between the empirical risk and the model complexity. The above formulation can be rewritten for non-linear case (Erasto 2001) as

$O(t)=\sum_{i=1}^{N_{s}} c_{i} \alpha_{i} K\left(t, s_{i}\right)+b$

where $s_{i}, i=1,2, \ldots . . N_{s}$ are support vectors that consist of a subset of $T$. The support vectors and coefficients are computed from $T$ via structural risk minimization. The Radial Basis Function (RBF) kernel is used in this study which is Gaussian in nature. RBF with window width of $\sigma$ (Erasto 2001) is defined as

$K(u, v)=\exp \left(-\frac{\|u-v\|^{2}}{\sigma^{2}}\right)$

More information about SVM can be found in (Cortes \& Vapnik 1995). The classification system is implemented with the help of dermoscopic images in $\mathrm{PH} 2$ database. This database comprises of three classes of dermoscopic images; normal, benign and malignant that requires a 3-class classification system. Though the original SVM classifier deals with binary (2-class) classification, it can be extended to multiclass classification by single machine methods (modifying the optimization problems) and decomposition strategies (one-vs-all and onevs-one techniques). However, these methods deal with larger optimization problem which is computationally more expensive (Rosales-Perez et al. 2018). Hence, a simple strategy of 2-phase classification (Thivya et al. 2016) is employed where the classification scenario in each phase is a 2-class problem.

In the $1^{\text {st }}$ phase, the available images in the PH2 database are grouped into two classes; one with negative labels that consists of normal dermoscopic images and another with positive 
labels which includes abnormal (benign and malignant) dermoscopic images. The task is to classify the given image as either normal or abnormal (NOAb) using SVM classifier (SVM-I in Figure 1). The performance of SVM model is validated using $k$-fold cross-validation.

The $k$-fold cross-validation procedure is as follows: At first, the dermoscopic images in the two classes (normal and abnormal) are randomly divided into $k$ groups such that the number of images in each fold is approximately equal. The images in one of the $k$-fold are treated as test set and the images in the remaining $k-1$ folds are treated as training set. The SVM classifier is trained using training set and then evaluated using the test set. The performance metrics are evaluated and retained for the particular $k$-fold. The same steps are repeated for all unique folds and then summarize the performance metrics of all folds. In machine learning, the commonly used k value is 10 (Ozkan \& Koklu 2017, Sonia 2016). Figure 2 shows the cross-validation procedure with 10 folds.

The next phase of classification is activated only when the result of $1^{\text {st }}$ phase classification is abnormal. In the $2^{\text {nd }}$ phase of classification (SVM-II), the benign dermoscopic images are

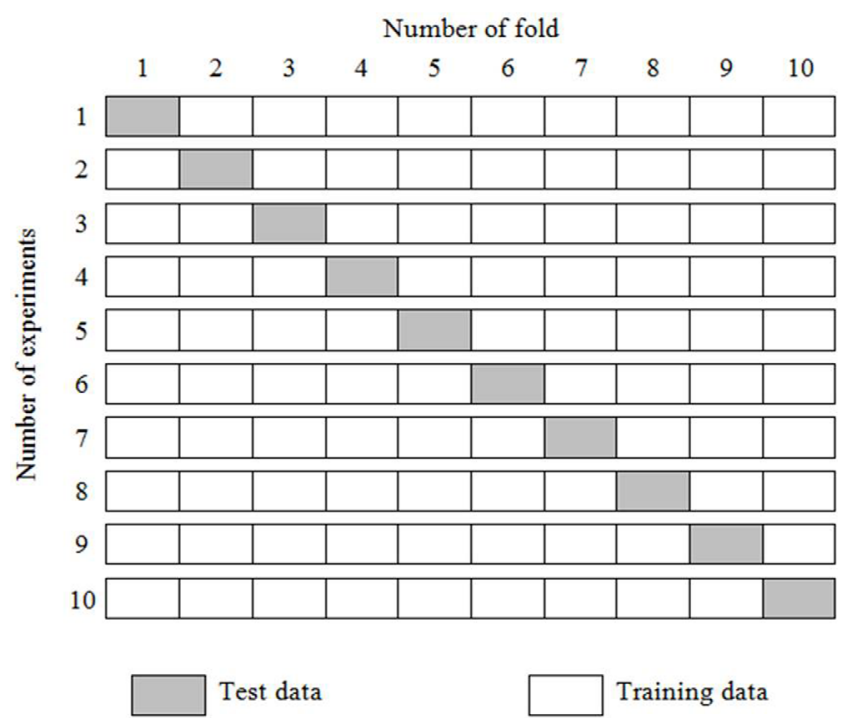

considered as one class with negative labels and malignant images as another class with positive labels. In this phase, the abnormal severity is again classified into benign or malignant (BeMa). The performance of the system at this phase is also evaluated using $k$-fold cross-validation which uses only benign and malignant dermoscopic images.

\section{PH2 Database}

The proposed skin cancer diagnostic system is analyzed using the $\mathrm{PH} 2$ database which is freely downloadable from (Mendonca et al. 2013 and PH2 database link). It contains 200 dermoscopic colour images with melanocytic lesions. The resolution of dermoscopic images is $768 \times 560$ pixels. There are 80 normal and 120 abnormal images available for the classification. Figure 3 shows sample normal and abnormal images in $\mathrm{PH} 2$ database.

\section{RESULTS AND DISCUSSIONS}

The performance of the system is validated using $k$-fold $(k=10)$ cross-validation and measured using accuracy, sensitivity, specificity and confusion matrix. Table I shows the basic

\section{Figure 2. Cross-validation procedure with 10 folds.}



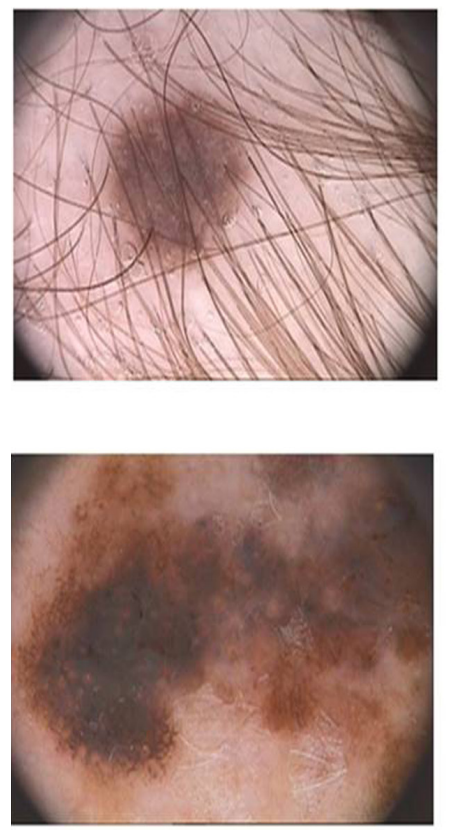
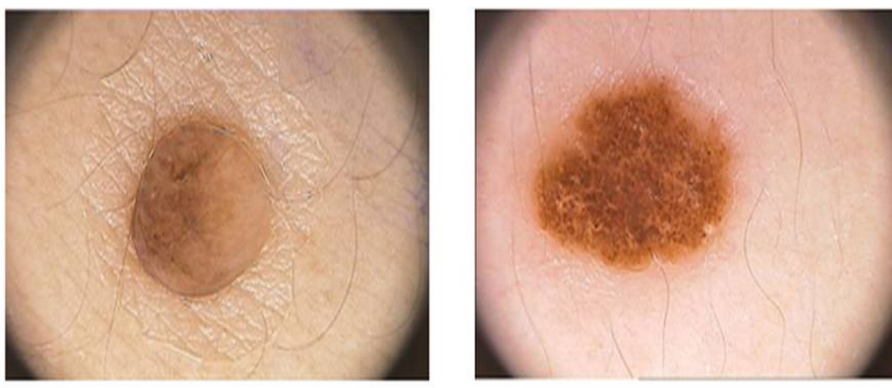

Figure 3. Sample images in the $\mathrm{PH} 2$ database (a) Normal images (b) melanoma images. (a)
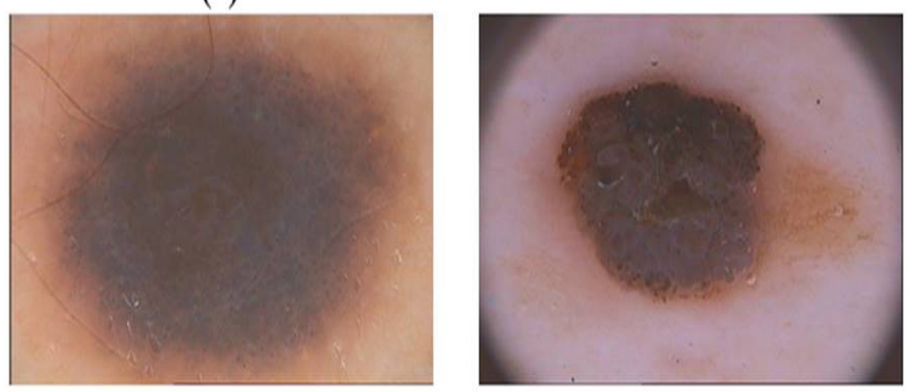

(b)

descriptions about the performance metrics used in this study.

In each phase of classification, the optimum value of kernel parameters $C$ and $\sigma$ which give maximum accuracy are found by grid search. It uses 10 different values of $C:\left[2^{7}, 2^{6}, 2^{5}, \ldots .2^{-2}\right]$ and $\sigma:\left[2^{4}, 2^{3}, 2^{2}, \ldots .2^{-5}\right]$. Hence, for each phase of classification $100(10 \times 10)$ combinations are tried. For each pair of $(C, \sigma)$, the validation performance is measured by 10 -fold crossvalidation. It is found that the best pair is $\left(2^{4}\right.$, $\left.2^{2}\right)$ for $1^{\text {st }}$ phase of classification and $\left(2^{7}, 2^{4}\right)$ for the $2^{\text {nd }}$ phase of classification. Tables II and III report the performances of the SCC system for the classification of the first phase and second phase respectively. The measurements in these tables are computed using the formulae in Table I. For a particular decomposition level (from 1 to 4), the BT sub-band features are extracted at different directions (2, 4, 8, 16 and 32). These features are classified using the 2-phase classification process.
It is observed from Table II that the $3^{\text {rd }}$ level BT features provide better performance than the features extracted at other levels. The maximum sensitivity, specificity and accuracy of the NOAb phase reach $97.5 \%, 100 \%$ and $98.5 \%$ respectively at 3 rd level BT with 8 directions. It is observed that the performance of SCC system decreases when the level of bendlet decomposition is above $3^{\text {rd }}$ level and the number of directional sub-bands for a particular decomposition level is above 8 directions. The highly redundant decomposition produces more number of subbands that do not have any discriminating power for the classification. The inclusion of these subbands features makes the classification system ineffective that reduces the system performance.

All the important performance metrics such as sensitivity, specificity and accuracy of BeMa phase reach $100 \%$ at $3^{\text {rd }}$ level BT with 8 directions. The obtained results demonstrate the effectiveness of BT as a feature extraction technique for the SCC system. A comparison of performance metrics with different image 
Table I. Performance matrices of SCC system.

\begin{tabular}{|c|c|c|}
\hline $\begin{array}{c}\text { Performance } \\
\text { measure }\end{array}$ & Description & Formula \\
\hline $\begin{array}{c}\text { Sensitivity } \\
\left(S_{n}\right)\end{array}$ & $\begin{array}{c}\text { It is the ratio between the number of } T_{r} P_{o} \text { decisions by } \\
\text { the classifier to the actual } P_{o} \text { cases tested. }\end{array}$ & $S_{n}=\frac{T_{r} P_{o}}{T_{r} P_{o}+F_{a} N_{e}}$ \\
\hline $\begin{array}{c}\text { Specificity } \\
\left(S_{p}\right)\end{array}$ & $\begin{array}{c}\text { It is the ratio between the number of } T_{r} N_{e} \text { decisions by } \\
\text { the classifier to the actual } N_{e} \text { cases tested. }\end{array}$ & $S_{p}=\frac{T_{r} N_{e}}{T_{r} N_{e}+F_{a} P_{o}}$ \\
\hline $\begin{array}{c}\text { Accuracy } \\
\left(A_{c}\right)\end{array}$ & $\begin{array}{c}\text { It is defined as the number of correctly classified skin } \\
\text { cancer images to the total number of images tested. }\end{array}$ & $A_{c}=\frac{T_{r} P_{o}+T_{r} N_{e}}{T_{r} P_{o}+F_{a} N_{e}+T_{r} N_{e}+F_{a} P_{o}}$ \\
\hline \multicolumn{2}{|c|}{$T_{r} P_{o} \rightarrow$ True Positive, $F_{a} N_{e} \rightarrow$ False Negative, $T_{r} N_{e} \rightarrow$ True Negative and $F_{a} P_{o} \rightarrow$ False Positive } \\
\hline
\end{tabular}

representation techniques is shown in Figures 4 and 5 for the NoAb phase and BeMa phase respectively.

It is observed from the performance comparisons of different image representation techniques in Figures 4 and 5 that BT performs well. It is obvious that wavelet transform is the least performer in this study as it provides less directional representation. Also, the performance of Contourlet is better than Curvelet as the approximations of Contourlet is more accurate than Curvelet.

The reason for the poor performance of wavelet is that they cannot characterize the boundary curves which separate different regions in dermoscopic images. These boundary curves are efficiently extracted by directional representation systems such as Curvelet and Contourlet which increases their performances over wavelet. The ability of Shearlet over Curvelet and Contourlet is the detection of non-smooth boundary curves between the image regions. BT is superior than Shearlet as it uses $\alpha$ scaling instead of parabolic scaling that helps to classify curvature precisely. Hence, the features extracted from the BT sub-bands are significant than other image representation systems.

Table IV shows the performance comparison of the system with existing approaches for SCC using PH2 dataset. As the system operates in 2-phases, the performance metrics shown in the table are the average performances of 2-phases in this system. It shows that the BT and SVM based system performed good as compare to existing SCC system in the literature.

\section{CONCLUSION}

In this paper, a framework for the SCC system based on BT and SVM classifier is presented. It includes directional representation based feature extraction and two SVM classifiers for classification. Compared to wavelets and Shearlets, BT can classify singularities in images more precisely. From the sub-bands of BT, energies are extracted. The 2-phase classification designed in this study classifies 
Table II. SVM-RBF Classifier performance for NoAb phase.

\begin{tabular}{|c|c|c|c|c|c|c|c|c|}
\hline \multirow{2}{*}{$\begin{array}{c}\text { Level of } \\
\text { Decomposition }\end{array}$} & \multirow{2}{*}{$\begin{array}{l}\text { Number of } \\
\text { directions }\end{array}$} & \multicolumn{7}{|c|}{ Performance Measures } \\
\hline & & $T_{r} P_{o}$ & $F_{a} N_{e}$ & $T_{r} N_{e}$ & $F_{a} P_{o}$ & $S_{n}(\%)$ & $S_{p}(\%)$ & $A_{c}(\%)$ \\
\hline \multirow{5}{*}{1} & 2 & 90 & 30 & 65 & 15 & 75.00 & 81.25 & 77.50 \\
\hline & 4 & 94 & 26 & 67 & 13 & 78.33 & 83.75 & 80.50 \\
\hline & 8 & 99 & 21 & 68 & 12 & 82.50 & 85.00 & 83.50 \\
\hline & 16 & 94 & 26 & 68 & 12 & 78.33 & 85.00 & 81.00 \\
\hline & 32 & 86 & 34 & 67 & 13 & 71.67 & 83.75 & 76.50 \\
\hline \multirow{5}{*}{2} & 2 & 97 & 23 & 70 & 10 & 80.83 & 87.50 & 83.50 \\
\hline & 4 & 102 & 18 & 72 & 8 & 85.00 & 90.00 & 87.00 \\
\hline & 8 & 108 & 12 & 75 & 5 & 90.00 & 93.75 & 91.50 \\
\hline & 16 & 100 & 20 & 71 & 9 & 83.33 & 88.75 & 85.50 \\
\hline & 32 & 93 & 27 & 71 & 9 & 77.50 & 88.75 & 82.00 \\
\hline \multirow{5}{*}{3} & 2 & 104 & 16 & 77 & 3 & 86.67 & 96.25 & 90.50 \\
\hline & 4 & 110 & 10 & 79 & 1 & 91.67 & 98.75 & 94.50 \\
\hline & 8 & 117 & 3 & 80 & 0 & 97.50 & 100.00 & 98.50 \\
\hline & 16 & 109 & 11 & 77 & 3 & 90.83 & 96.25 & 93.00 \\
\hline & 32 & 102 & 18 & 75 & 5 & 85.00 & 93.75 & 88.50 \\
\hline \multirow{5}{*}{4} & 2 & 97 & 23 & 79 & 1 & 80.83 & 98.75 & 88.00 \\
\hline & 4 & 108 & 12 & 79 & 1 & 90.00 & 98.75 & 93.50 \\
\hline & 8 & 110 & 10 & 79 & 1 & 91.67 & 98.75 & 94.50 \\
\hline & 16 & 104 & 16 & 79 & 1 & 86.67 & 98.75 & 91.50 \\
\hline & 32 & 96 & 24 & 78 & 2 & 80.00 & 97.50 & 87.00 \\
\hline
\end{tabular}


Table III. SVM-RBF Classifier performance for BeMa phase.

\begin{tabular}{|c|c|c|c|c|c|c|c|c|}
\hline \multirow{2}{*}{$\begin{array}{c}\text { Level of } \\
\text { Decomposition }\end{array}$} & \multirow{2}{*}{$\begin{array}{l}\text { Number of } \\
\text { directions }\end{array}$} & \multicolumn{7}{|c|}{ Performance Measures } \\
\hline & & $T_{r} P_{o}$ & $F_{a} N_{e}$ & $T_{r} N_{e}$ & $F_{a} P_{o}$ & $S_{n}(\%)$ & $S_{p}(\%)$ & $A_{c}(\%)$ \\
\hline \multirow{5}{*}{1} & 2 & 34 & 6 & 67 & 13 & 85.00 & 83.75 & 84.17 \\
\hline & 4 & 35 & 5 & 69 & 11 & 87.50 & 86.25 & 86.67 \\
\hline & 8 & 36 & 4 & 74 & 6 & 90.00 & 92.50 & 91.67 \\
\hline & 16 & 34 & 6 & 72 & 8 & 85.00 & 90.00 & 88.33 \\
\hline & 32 & 34 & 6 & 63 & 17 & 85.00 & 78.75 & 80.83 \\
\hline \multirow{5}{*}{2} & 2 & 36 & 4 & 70 & 10 & 90.00 & 87.50 & 88.33 \\
\hline & 4 & 37 & 3 & 74 & 6 & 92.50 & 92.50 & 92.50 \\
\hline & 8 & 39 & 1 & 77 & 3 & 97.50 & 96.25 & 96.67 \\
\hline & 16 & 36 & 4 & 74 & 6 & 90.00 & 92.50 & 91.67 \\
\hline & 32 & 33 & 7 & 72 & 8 & 82.50 & 90.00 & 87.50 \\
\hline \multirow{5}{*}{3} & 2 & 38 & 2 & 71 & 9 & 95.00 & 88.75 & 90.83 \\
\hline & 4 & 38 & 2 & 77 & 3 & 95.00 & 96.25 & 95.83 \\
\hline & 8 & 40 & 0 & 80 & 0 & 100.00 & 100.00 & 100.00 \\
\hline & 16 & 37 & 3 & 75 & 5 & 92.50 & 93.75 & 93.33 \\
\hline & 32 & 36 & 4 & 72 & 8 & 90.00 & 90.00 & 90.00 \\
\hline \multirow{5}{*}{4} & 2 & 33 & 7 & 74 & 6 & 82.50 & 92.50 & 89.17 \\
\hline & 4 & 37 & 3 & 76 & 4 & 92.50 & 95.00 & 94.17 \\
\hline & 8 & 39 & 1 & 78 & 2 & 97.50 & 97.50 & 97.50 \\
\hline & 16 & 36 & 4 & 75 & 5 & 90.00 & 93.75 & 92.50 \\
\hline & 32 & 32 & 8 & 74 & 6 & 80.00 & 92.50 & 88.33 \\
\hline
\end{tabular}

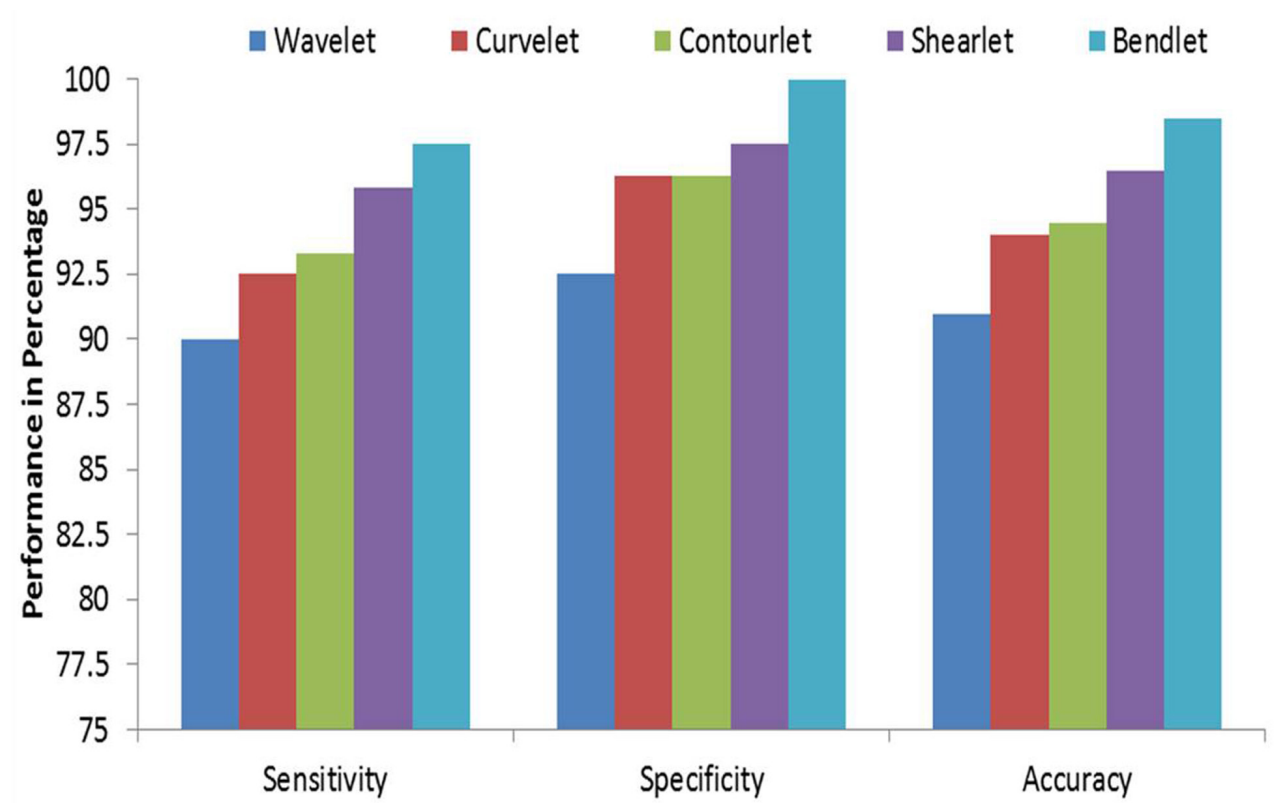

Figure 4. Performance comparisons of different image representation techniques for the classification of NoAb phase. 


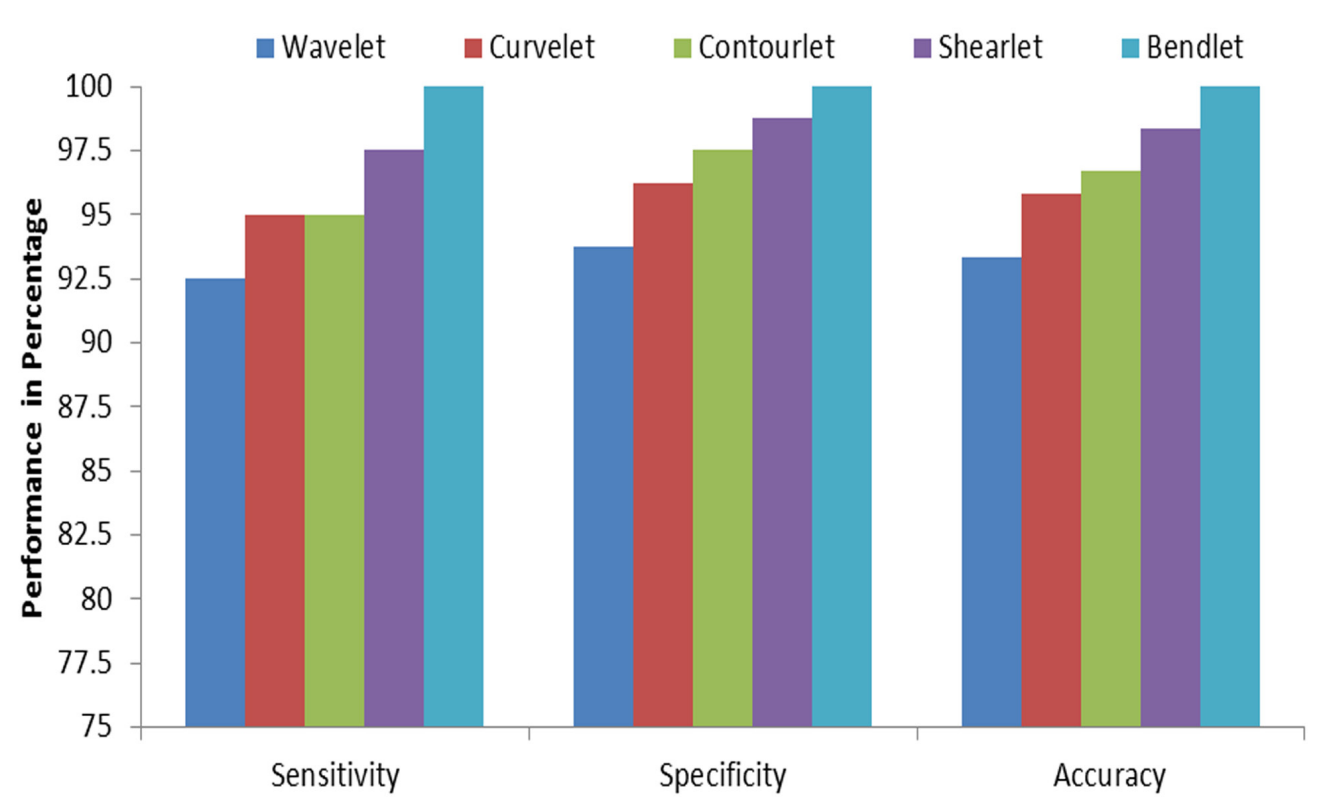

Figure 5.

Performance comparisons of different image representation techniques for the classification of BeMa phase.

Table IV. Comparison of BT and SVM based SCC system results with existing systems.

\begin{tabular}{|c|c|c|c|c|}
\hline Author & \#Images used & Accuracy (\%) & Sensitivity (\%) & Specificity (\%) \\
\hline Ozkan \& Koklu (2017) & 200 & 92.50 & 90.86 & 96.11 \\
\hline Zaqout (2016) & 200 & 90 & 85 & 92.22 \\
\hline Sonia (2016) & 120 & 96.7 & 97.5 & 96.3 \\
\hline Nasir et al. (2018) & 150 & 97.50 & 97.70 & 96.70 \\
\hline Proposed system & $\mathbf{2 0 0}$ & $\mathbf{9 8 . 7 5}$ & $\mathbf{9 9 . 2 5}$ & $\mathbf{1 0 0}$ \\
\hline
\end{tabular}

the dermoscopic images into normal/abnormal and then cancerous/noncancerous. Results show that the SCC system using BT and SVM classifier provides better accuracy, specificity and sensitivity over wavelets and Shearlets. Also, the SCC system does not require any manual intervention for the diagnosis. The validation of the SCC system on a larger database will be done in the near future.

\section{Abbreviations}

- SCC - Skin Cancer Classification

- BT - Bendlet Transform

- SVM - Support Vector Machine
- $\quad A B C D$ - Asymmetry, Border, Colour and Diameter

- NoAb - Normal or Abnormal

- BeMa - Benign or Malignant

- $\mathrm{S}_{\mathrm{n}}$ - Sensitivity

- $S_{p}$ - Specificity

- $A_{c}$ - Accuracy

- $\mathrm{T}_{\mathrm{r}} \mathrm{P}_{0}$ - True Positive

- $\mathrm{F}_{\mathrm{a}} \mathrm{N}_{\mathrm{e}}$ - False Negative

- $\quad \mathrm{T}_{\mathrm{r}} \mathrm{N}_{\mathrm{e}}$ - True Negative

- $\quad \mathrm{F}_{\mathrm{a}} \mathrm{P}_{0}$ - False Positive

\section{REFERENCES}

ABU MAHMOUD M, AL-JUMAILY A \& TAKRURI MS. 2013. Wavelet and curvelet analysis for automatic identification of 
melanoma based on neural network classification. IJCISIM 5: 606-614.

ARIAS-CASTRO E \& DONOHO DL. 2009. Does median filtering truly preserve edges better than linear filtering? Ann Stat 37(3): 1172-1206.

ALI AJ \& JANET J. 2013. MaSS classification in digital mammograms based on discrete shearlet transform. JCS 9(6): 726-732.

ALMANSOUR E \& JAFFAR MA. 2016. Classification of Dermoscopic skin cancer images using colour and hybrid texture features. IJCSNS 16(4): 135-139.

CORTES C \& VAPNIK V. 1995. Support-vector networks. Machine learning 20(3): 273-297.

DE VITA V, DI LEO GD, FABBROCINI G, LIGUORI C, PAOLILLO A \& SOMMELLA P. 2012. Statistical techniques applied to the automatic diagnosis of dermoscopic images. Acta Imeko 1(1): 7-18.

DO MN \& VETTERLI M. 2005. The contourlet transform: an efficient directional multiresolution image representation. IEEE Trans Image Process 14(12): 2091-2106.

DONOHO D \& CANDES E. 2005. Continuous curvelet transform: II. Discretization and frames. ACHA 19(2): 198-222.

ERASTO P. 2001. Support vector machines-backgrounds and practice, Academic Dissertation for The Degree of Licentiate of Philosophy. Rolf Nevanlinna Institute, Helsinki.

ESTEVA A, KUPREL B, NOVOA RA, KO J, SWETTER SM, BLAU HM \& THRUN S. 2017. Dermatologist-level classification of skin cancer with deep neural networks. Nature 542(7639): 115-118.

JAIN YK \& JAIN M. 2012. Skin cancer detection and classification using Wavelet Transform and Probabilistic Neural Network. In: $4^{\text {th }}$ International Conference on ARTCom, Bangalore, India, p. 250-252.

LESSIG C, PETERSEN P \& SCHÄFER BENDLETS M. 2017. A secondorder shearlet transform with bent elements. ACHA 46(2): 384-399.

LIM WQ. 2010. The discrete shearlet transform: a new directional transform and compactly supported shearlet frames. IEEE Trans Image Process 19(5): 1166-1180.

MA Z \& TAVARES JM. 2017. Effective features to classify skin lesions in dermoscopic images. Expert Syst Appl 84: 92-101.
MALLAT S. 2008. A wavelet tour of signal processing: the sparse way. Academic Press.

MENDONÇA T, FERREIRA PM, MARQUES JS, MARCAL AR \& ROZEIRA J. 2013. PH2 - A dermoscopic image database for research and benchmarking. In: $35^{\text {th }}$ Annual International Conference On EMBS, Osaka, Japan, p. 5437-5440.

NASIR M, ATTIQUE KHAN M, SHARIF M, LALI IU, SABA T \& IQBAL T. 2018. An improved strategy for skin lesion detection and classification using uniform segmentation and feature selection based approach. MRT 81(6): 528-543.

OZKAN IA \& KOKLU M. 2017. Skin Lesion Classification using Machine Learning Algorithms. IJISAE 5(4): 285-289.

PH2 Database Link: https://www.fc.up.pt/addi/ph2\%20 database.html

ROSALES-PEREZ A, GARCIA S, TERASHIMA-MARIN H, COELLO CA \& HERRERA F. 2018. MC ${ }^{2}$ ESVM: Multiclass classification based on cooperative evolution of support vector machines. IEEE CIM 13(2): 18-29.

RUIZ D, BERENGUER V, SORIANO A \& SÁNCHEZ B. 2011. A decision support system for the diagnosis of melanoma: A comparative approach. Expert Syst Appl 38(12): 15217-15223.

SELVATHI D, PRAKASH NB, GOMATHI V \& HEMALAKSHMI GR. 2018. Fundus Image Classification Using Wavelet-Based Features in Detection of Glaucoma. BPJ 11(2): 795-805.

SONIA R. 2016. Melanoma image classification system by NSCT features and Bayes classification. IJASIS 2(2): 27-33.

THIVYA KS, SAKTHIVEL P \& VENKATA SAI PM. 2016. Analysis of framelets for breast cancer diagnosis. THC 24(1): 21-29.

WANG ZZ \& YONG JH. 2008. Texture analysis and classification with linear regression model based on the wavelet transform. IEEE Trans Image Process 17(8): 1421-1430.

ZAQOUT I. 2016. Diagnosis of skin lesions based on dermoscopic images using image processing techniques. IJSIP 9(9): 189-204.

\section{How to cite}

POOVIZHI S \& GANESH BABU TR 2020. An Efficient Skin Cancer Diagnostic System Using Bendlet Transform and Support Vector Machine. An Acad Bras Cienc 92: e20190554. DOI 10.1590/0001-3765202020190554.

\section{POOVIZHI S ${ }^{1}$}

https://orcid.org/0000-0003-1862-1908

\section{GANESH BABU TR ${ }^{2}$}

https://orcid.org/0000-0002-1806-4323 
${ }^{1}$ Anna University, Department of Electronics and

Communication Engineering, Tamilnadu-600025, India

${ }^{2}$ Muthayammal Engineering College,

Department of Electronics and Communication Engineering,

Tamilnadu-637408, India

Manuscript received on May 20, 2019;

accepted for publication on August 5, 2019

Correspondence to: Poovizhi S

E-mail:poovizhiece22@gmail.com

\section{Author contributions}

Poovizhi S conducted the study by reviewing existing systems, collecting data, performing the simulations in MATLAB, and producing the results. Also, Poovizhi S drafted the paper. Ganesh Babu TR supervised the research, performed the statistical analysis and contributed to the discussion. All authors revised and reviewed the manuscript.

\section{(cc) BY}

